\title{
A Critical Review on the Complex Potentials in Linear Elastic Fracture Mechanics
}

\author{
Johannes Scheel $^{1}$ (D) Daniel Wallenta ${ }^{2} \cdot$ Andreas Ricoeur $^{1}$
}

Received: 17 February 2021 / Accepted: 9 December 2021 / Published online: 3 January 2022

(c) The Author(s) 2022, corrected publication 2022

\begin{abstract}
Introducing a crack in an elastic plate is challenging from the mathematical point of view and relevant within an engineering context of evaluating strength and reliability of structures. Accordingly, a multitude of associated works is available to date, emanating from both applied mathematics and mechanics communities. Although considering the same problem, the given complex potentials prove to be different, revealing various inconsistencies in terms of resulting stresses and displacements. Essential information on crack near-tip fields and crack opening displacements is nonetheless available, while intuitive adaption is required to obtain the full-field solutions. Investigating the cause of prevailing deficiencies inevitably leads to a critical review of classical works by Muskhelishvili or Westergaard. Complex potentials of the mixed-mode loaded Griffith crack, sparing restrictive assumptions or limitations of validity, are finally provided, allowing for rigorous mathematical treatment. The entity of stresses and displacements in the whole plate is finally illustrated and the distributions in the crack plane are given explicitly.
\end{abstract}

Keywords Complex potentials · Westergaard stress function · Holomorphic functions · Griffith crack · Kolosov's equations · Crack fields

Mathematics Subject Classification (2010) 74B05 · 74R10 · 30A99

\section{Introduction}

With the goal of calculating strains at every point in a beam, Airy introduced the now called Airy stress function [1]. With this function, the constitutive law of elasticity and compatibility conditions of strains a biharmonic equation is derived, finally providing stress solutions if an appropriate ansatz of the Airy stress function is chosen, satisfying boundary conditions. The first work on elastic crack problems is commonly attributed to Inglis [13], who adopted Love's solution of a plate with an elliptic hole [18] as a basis. Already six years before,

$\triangle$ J. Scheel

j.scheel@uni-kassel.de

1 Institute of Mechanics, University of Kassel, 34125 Kassel, Germany

2 Institute of Mathematics, University of Kassel, 34109 Kassel, Germany 
Wieghardt [34], however, published a paper in German language, translated "On the cleavage and rupture of elastic bodies", where stresses at cracks and sharp corners are rigorously derived from Airy's stress function.

A more general and contemporary solution of modern fracture mechanics employs complex function theory and is given by two complex holomorphic potentials. Kolosov [15] was the first to obtain the displacements and stresses based on these two functions. Muskhelishvili [20] later extensively utilized these relations and comprehensively investigated the holomorphic functions, being the basis of a very general and powerful approach to determine the solution of various boundary value problems. In fracture mechanics, this approach flourished as it enables the calculation of stress and displacement fields in spite of noncontinuous and non-smooth boundary conditions along the crack plane, finally allowing for the calculation of the crack tip loading.

A less general but more common approach has subsequently been introduced by Westergaard [33] in terms of the Westergaard stress functions which is also applied to determine stress and displacement fields. However, the originally introduced approach was deemed incomplete, only solving a restricted class of crack problems, but was extended to resolve these restrictions [25, 27, 29]. For particular classes of problems, the two-complex-potential approach can be simplified to an approach requiring only one function, exploiting certain symmetries [27]. This single complex potential is related to the Westergaard stress function. The two methods are widespread in fracture mechanics featuring a multitude of references applied to various crack problems. A lot of them have been compiled, e.g., by Tada et al. [31].

For the Griffith crack, as the most simple configuration, sparing boundary conditions apart from the crack faces being embedded in an infinite elastic plate, numerous authors, e.g., [2, 4, 6, 8-10, 12, 14, 16, 17, 19, 20, 22, 23, 26-28, 30-33, 35, 37], provide complex potentials or stress functions for mode $I$ loading. Much less authors also provide those for mode II loading [2, 4, 9, 16, 19, 20, 23, 27, 30, 31]. The majority of references in this context either cite Muskhelishvili or Westergaard. While Muskhelishvili's functions for the Griffith crack are predominantly adopted correctly, Westergaard's original stress function, however, is scarcely transcribed appropriately.

However, Muskhelishvili's complex potentials (e.g., [4, 6, 8, 10, 12, 16, 19, 20, 23, 26, 27]) and the Westergaard stress functions as mostly employed (e.g., [2, 9, 12, 17, 22, 28, 30$32,35,37])$ are just strictly valid in the positive $(x>0)$ half plane of the crack, see hatched area in Fig. 1. Applying them to the whole domain, obviously reveals discontinuities and unphysical point symmetry. Stresses and displacements in the negative half-plane are thus, inter alia, either obtained intuitively by reflection in the $y$-axis or by adapting the equations introducing an a priori case-by-case analysis which, however, is not mathematically motivated. While the limited validity in [20] is due to a transformation in the complex number plane which will be discussed in Sect. 4, the approach presented in [33] is basically valid on the whole domain, however, has been transferred erroneously to contemporary literature, basically giving rise to the same issue as in [20].

Following a brief presentation of essential fundamentals in Sect. 2, the complex potentials and stress functions provided by established literature as well as resulting stress and displacement fields are discussed in Sect. 3. Due to various conspicuous features in the results, works of Muskhelishvili [20] and Westergaard [33] and their transfer to contemporary literature are critically reviewed. In this context, Sect. 4 provides details on some mathematical issues to be considered. Subsequently, in Sect. 5, current approaches to overcome deficiencies and complement stress and displacement solutions are outlined, finally illuminating why this problem did not attract attention yet. Complex potentials for the whole elastic domain of the Griffith crack are eventually presented in Sect. 6, avoiding case-by-case 
Fig. 1 Mode-I/II loaded Griffith crack; hatched area indicates the scope of validity of commonly available solutions

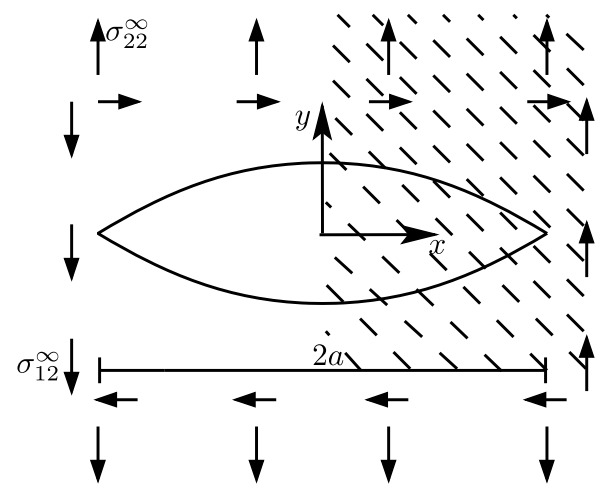

analysis and incorporating rigid body rotation for the sake of generality, which is basically disregarded in literature. The crack fields are calculated sparing ineligible discontinuities or other irregularities. Being of major interest in fracture mechanics, all stresses and displacements in the crack plane are given explicitly.

\section{Some Fundamentals of Elastic Crack Solutions}

An arbitrarily loaded straight crack of the length $2 a$ in an infinitely large sheet of isotropic linear-elastic material, i.e., a Griffith crack, is depicted in Fig. 1. The origin of the complex plane is located in the center of the crack, introducing the complex coordinate $z=x+\mathrm{i} y$. The positions of the crack faces are defined as:

$$
-a \leq x \leq a, \quad y= \pm 0
$$

assuming traction-free boundaries, i.e.,

$$
\sigma_{12}^{ \pm}(x)=0, \quad \sigma_{22}^{ \pm}(x)=0 \quad \text { for } \quad|x|<a,
$$

where the superscript \pm indicates the positive $(+)$ or negative $(-)$ crack face. The stresses at infinity are controlled by uniaxial tension $\sigma_{22}^{\infty}$ and in-plane shear loading $\sigma_{12}^{\infty}$ :

$$
\sigma_{22}(z)=\sigma_{22}^{\infty}, \quad \sigma_{12}(z)=\sigma_{12}^{\infty}, \quad \sigma_{11}(z)=\sigma_{11}^{\infty}=0 \quad \text { for } \quad|z|=\sqrt{x^{2}+y^{2}} \rightarrow \infty
$$

The calculation of the crack fields is achieved by Kolosov's equations [15]

$$
\begin{aligned}
& \sigma_{11}(z)+\sigma_{22}(z)=2\left(\Phi^{\prime}(z)+\overline{\Phi^{\prime}(z)}\right)=4 \operatorname{Re}\left[\Phi^{\prime}(z)\right], \\
& \sigma_{22}(z)-\sigma_{11}(z)+2 \mathrm{i} \sigma_{12}(z)=2\left(\bar{z} \Phi^{\prime \prime}(z)+\Psi^{\prime}(z)\right), \\
& 2 \mu\left(u_{1}(z)+\mathrm{i} u_{2}(z)\right)=\kappa \Phi(z)-z \overline{\Phi^{\prime}(z)}-\overline{\Psi(z)},
\end{aligned}
$$

where $\Phi$ and $\Psi$ are holomorphic complex potentials on $\mathbb{C} \backslash[-a, a]$ with their respective spatial derivatives $\Phi^{\prime}, \Phi^{\prime \prime}$ and $\Psi^{\prime}$. The stresses are denoted as $\sigma_{i j}$ and $u_{i}$ are the displacements, where $\mu$ is the shear modulus and $\kappa$ is Kolosov's parameter. Bars on quantities denote 
complex conjugates, e.g., $\bar{z}=x-\mathrm{i} y$. For holomorphic functions, the Cauchy-Riemann equations are satisfied and employing the Wirtinger derivatives [36], the displacement gradients are calculated as:

$$
\begin{aligned}
& \frac{\partial u_{1}(z)}{\partial x}=\frac{1}{2 \mu} \operatorname{Re}\left[\kappa \Phi^{\prime}(z)-\overline{\Phi^{\prime}(z)}-z \overline{\Phi^{\prime \prime}(z)}-\overline{\Psi^{\prime}(z)}\right], \\
& \frac{\partial u_{1}(z)}{\partial y}=\frac{1}{2 \mu} \operatorname{Re}\left[\kappa \mathrm{i} \Phi^{\prime}(z)-\mathrm{i} \overline{\Phi^{\prime}(z)}+z \overline{\mathrm{i}} \overline{\Phi^{\prime \prime}(z)}+\mathrm{i} \overline{\Psi^{\prime}(z)}\right], \\
& \frac{\partial u_{2}(z)}{\partial x}=\frac{1}{2 \mu} \operatorname{Im}\left[\kappa \Phi^{\prime}(z)-\overline{\Phi^{\prime}(z)}-z \overline{\Phi^{\prime \prime}(z)}-\overline{\Psi^{\prime}(z)}\right], \\
& \frac{\partial u_{2}(z)}{\partial y}=\frac{1}{2 \mu} \operatorname{Im}\left[\kappa \mathrm{i} \Phi^{\prime}(z)-\mathrm{i} \overline{\Phi^{\prime}(z)}+z \overline{\mathrm{i}} \overline{\Phi^{\prime \prime}(z)}+\mathrm{i} \overline{\Psi^{\prime}(z)}\right] .
\end{aligned}
$$

Exploiting the axial and skew symmetries for the mode I and II, respectively, loading of the Griffith crack, the two complex potentials can be reduced to one, requiring a relation between $\Phi$ and $\Psi$ [27]. According to [25, 29], functions $A(z)$ and $B(z)$ have to be introduced for the sake of generality, whereupon

$$
\begin{aligned}
& \Psi_{I}^{\prime}(z)=-z \Phi_{I}^{\prime \prime}(z)-A \\
& \Psi_{I I}^{\prime}(z)=-2 \Phi_{I I}^{\prime}(z)-z \Phi_{I I}^{\prime \prime}(z)-\mathrm{i} B
\end{aligned}
$$

with real constants $A$ and $B$ is provided as special case in [27, 29] being valid for the considered problem. The subscript $I$ or $I I$ indicates the affiliation to the respective crack opening mode. Furthermore, the reduction to one complex potential enables a relation to the Westergaard stress functions [33] $Z_{I}(z), Z_{I I}(z)$ according to [25, 29]:

$$
\begin{aligned}
& \Phi_{I}^{\prime}(z)=\frac{1}{2}\left(Z_{I}(z)+A\right), \quad \Psi_{I}^{\prime}(z)=-\frac{1}{2}\left(z Z_{I}^{\prime}(z)\right)-A \\
& \Phi_{I I}^{\prime}(z)=-\frac{\mathrm{i}}{2}\left(Z_{I I}(z)+B\right), \quad \Psi_{I I}^{\prime}(z)=\mathrm{i} Z_{I I}(z)+\frac{\mathrm{i} z}{2}\left(Z_{I I}^{\prime}(z)\right) .
\end{aligned}
$$

Applying the superposition principle, e.g., $\Phi^{\prime}=\Phi_{I}^{\prime}+\Phi_{I I}^{\prime}$, and inserting Eq. (7) into Eq. (4) yields

$$
\begin{aligned}
\sigma_{11}(z)= & \operatorname{Re}\left[Z_{I}(z)\right]-y \operatorname{Im}\left[Z_{I}^{\prime}(z)\right]+2 A+2 \operatorname{Im}\left[Z_{I I}(z)\right]+y \operatorname{Re}\left[Z_{I I}^{\prime}(z)\right], \\
\sigma_{22}(z)= & \operatorname{Re}\left[Z_{I}(z)\right]+y \operatorname{Im}\left[Z_{I}^{\prime}(z)\right]-y \operatorname{Re}\left[Z_{I I}^{\prime}(z)\right], \\
\sigma_{12}(z)= & -y \operatorname{Re}\left[Z_{I}^{\prime}(z)\right]+\operatorname{Re}\left[Z_{I I}(z)\right]-y \operatorname{Im}\left[Z_{I I}^{\prime}(z)\right] \\
2 \mu u_{1}(z)= & \frac{\kappa-1}{2} \operatorname{Re}\left[\hat{Z}_{I}(z)\right]-y \operatorname{Im}\left[Z_{I}(z)\right]+\frac{\kappa+1}{2} A x+\frac{\kappa+1}{2} \operatorname{Im}\left[\hat{Z}_{I I}(z)\right] \\
& +y \operatorname{Re}\left[Z_{I I}(z)\right]+\frac{\kappa+1}{2} B y, \\
2 \mu u_{2}(z)= & \frac{\kappa+1}{2} \operatorname{Im}\left[\hat{Z}_{I}\right]-y \operatorname{Re}\left[Z_{I}(z)\right]+\frac{\kappa-3}{2} A y-\frac{\kappa-1}{2} \operatorname{Re}\left[\hat{Z}_{I I}(z)\right] \\
& -y \operatorname{Im}\left[Z_{I I}(z)\right]-\frac{\kappa+1}{2} B x
\end{aligned}
$$


with $Z_{I / I I}$ being the spatial derivative of $\hat{Z}_{I / I I}$. The constant $B$ has an impact just on the displacements and $A$ does not contribute to singular crack tip stresses, accounting for, inter alia, homogeneous stress in case of tension or compression loading in $x$-direction.

\section{Commonly Applied Complex Potentials}

The complex potentials commonly employed for the Griffith crack are basically given in $[4,16,23,27]$, whereas $[6,8,10,12,26]$ only cover mode I. Providing just $\Phi$ and $\Psi$ or $\Phi^{\prime}$ and $\Psi^{\prime}$, and sometimes only $\Phi$ or $\Phi^{\prime}$ bearing on Eq. (6), some calculus is required to obtain the whole set of functions to be inserted into Eqs. (4) and (5). Evolving integration constants represent rigid body translation and may be canceled in a local crack related coordinate system, which is mentioned briefly only in [6]. Rigid body rotation, being included in the derivatives of the potentials, is basically disregarded, thus depriving the solution of some generality. In $[19,20]$ this issue is taken into account, however, skipped in an early stage of derivation. Following [27], in which $\Phi^{\prime}$ and $\Psi^{\prime}$ are given, and assuming $\sigma_{11}^{\infty}=0$ the complex potentials read:

$$
\begin{aligned}
& \Phi(z)=\frac{\sigma_{22}^{\infty}}{2} \sqrt{z^{2}-a^{2}}-\frac{\sigma_{22}^{\infty}}{4} z+\mathrm{i} \frac{\sigma_{12}^{\infty}}{2} z-\mathrm{i} \frac{\sigma_{12}^{\infty}}{2} \sqrt{z^{2}-a^{2}}, \\
& \Phi^{\prime}(z)=\frac{\sigma_{22}^{\infty}}{2} \frac{z}{\sqrt{z^{2}-a^{2}}}-\frac{\sigma_{22}^{\infty}}{4}+\mathrm{i} \frac{\sigma_{12}^{\infty}}{2}-\mathrm{i} \frac{\sigma_{12}^{\infty}}{2} \frac{z}{\sqrt{z^{2}-a^{2}}}, \\
& \Phi^{\prime \prime}(z)=-\frac{\sigma_{22}^{\infty}}{2} \frac{a^{2}}{\left(z^{2}-a^{2}\right)^{\frac{3}{2}}}+\mathrm{i} \frac{\sigma_{12}^{\infty}}{2} \frac{a^{2}}{\left(z^{2}-a^{2}\right)^{\frac{3}{2}}}, \\
& \Psi(z)=\frac{\sigma_{22}^{\infty}}{2} z-\frac{\sigma_{22}^{\infty}}{2} \frac{a^{2}}{\sqrt{z^{2}-a^{2}}}+\frac{\mathrm{i} \sigma_{12}^{\infty}}{2} \frac{2 z^{2}-a^{2}}{\sqrt{z^{2}-a^{2}}}, \\
& \Psi^{\prime}(z)=\frac{\sigma_{22}^{\infty}}{2}+\frac{\sigma_{22}^{\infty}}{2} \frac{z a^{2}}{\left(z^{2}-a^{2}\right)^{\frac{3}{2}}}+\frac{\mathrm{i} \sigma_{12}^{\infty}\left(2 z^{3}-3 z a^{2}\right)}{2\left(z^{2}-a^{2}\right)^{\frac{3}{2}}} .
\end{aligned}
$$

In [16] a different fraction $+\mathrm{i} \sigma_{12}^{\infty} / 4$ is found for the constant mode II term in $\Phi^{\prime}$, in [23] $-\sigma_{22}^{\infty} / 2$ is given for the mode I term. Inserted into Eq. (4), Eq. (9) provides the crack fields depicted in Figs. 2 and 3. The figures obviously reveal inconsistencies, e.g., the crack tip stress singularities of $\sigma_{22}$ and $\sigma_{11}$ in mode I and $\sigma_{12}$ in mode II exhibit point symmetry and the displacement field is discontinuous ( $u_{2}$ of mode $\mathrm{I}, u_{1}$ of mode II) on $\mathbb{C} \backslash[-a, a]$. Accordingly, the equations of Eq. (9), commonly denoted as holomorphic functions, do not meet the requirements of this specification.

Complex potentials were extensively elaborated by Muskhelishvili [20], originally introducing $\Phi^{\prime}$ and $\Psi^{\prime}$ according to

$$
\begin{aligned}
& \Phi^{\prime}(z)=\frac{\left(2 \Gamma+\overline{\Gamma_{2}}\right) z}{2 \sqrt{z^{2}-a^{2}}}-\frac{1}{2} \overline{\Gamma_{2}}, \\
& \Psi^{\prime}(z)=\overline{\Omega(\bar{z})}-\Phi^{\prime}(z)-z \Phi^{\prime \prime}(z), \\
& \Omega(z)=\frac{\left(2 \Gamma+\overline{\Gamma_{2}}\right) z}{2 \sqrt{z^{2}-a^{2}}}+\frac{1}{2} \overline{\Gamma_{2}},
\end{aligned}
$$



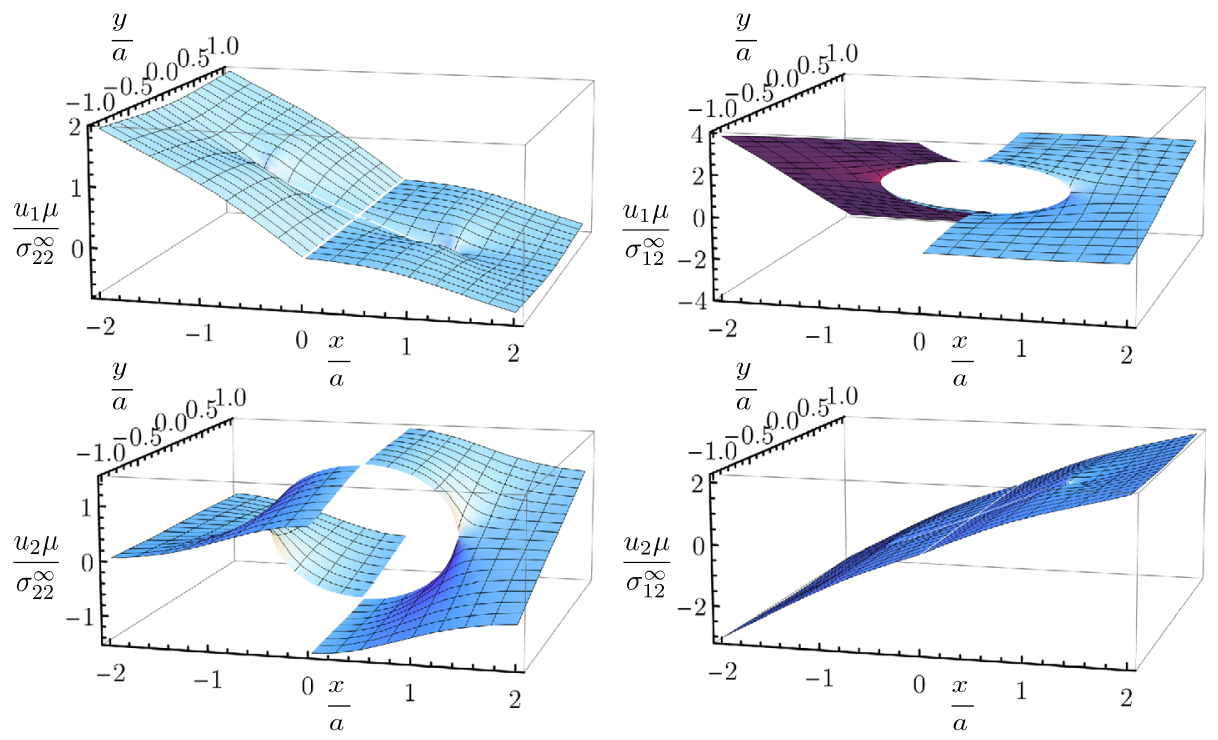

Fig. 2 Normalized displacement fields for mode I (left) and mode II (right) for a Griffith crack calculated with the complex potentials of Eq. (9)

with

$$
\begin{aligned}
& \Gamma=\frac{1}{4}\left(\sigma_{I}+\sigma_{I I}\right), \\
& \Gamma_{2}=-\frac{1}{2}\left(\sigma_{I}-\sigma_{I I}\right) \mathrm{e}^{-2 \mathrm{i} \alpha} .
\end{aligned}
$$

In general

$$
\Gamma=\frac{1}{4}\left(\sigma_{I}+\sigma_{I I}\right)+\mathrm{i} \frac{2 \mu \varepsilon_{\infty}}{1+\kappa}
$$

holds, where $\varepsilon_{\infty}$ is the rigid body rotation at infinity which is set to zero in the derivation of Eq. (10) in [20]. Furthermore, $\sigma_{I / I I}$ are the principal stresses at infinity and $\alpha$ is the angle between the $\sigma_{I^{-}}$and the $x$-axis. Equations (10) and (11) are cited by, e.g., [7, 32]. For the case of biaxial tensile and in-plane shear stress loading, $\Gamma$ and $\Gamma_{2}$ read

$$
\begin{aligned}
& \Gamma=\frac{\sigma_{22}^{\infty}}{4}+\frac{\sigma_{11}^{\infty}}{4}, \\
& \Gamma_{2}=\frac{\sigma_{22}^{\infty}}{2}-\frac{\sigma_{11}^{\infty}}{2}+\mathrm{i} \sigma_{12}^{\infty},
\end{aligned}
$$

inserted into Eq. (10) resulting in the functions of Eq. (9), for $\sigma_{11}^{\infty}=0$. It is noted that in the original [20] $\Gamma_{2}$ is denoted as $\Gamma^{\prime}$. 

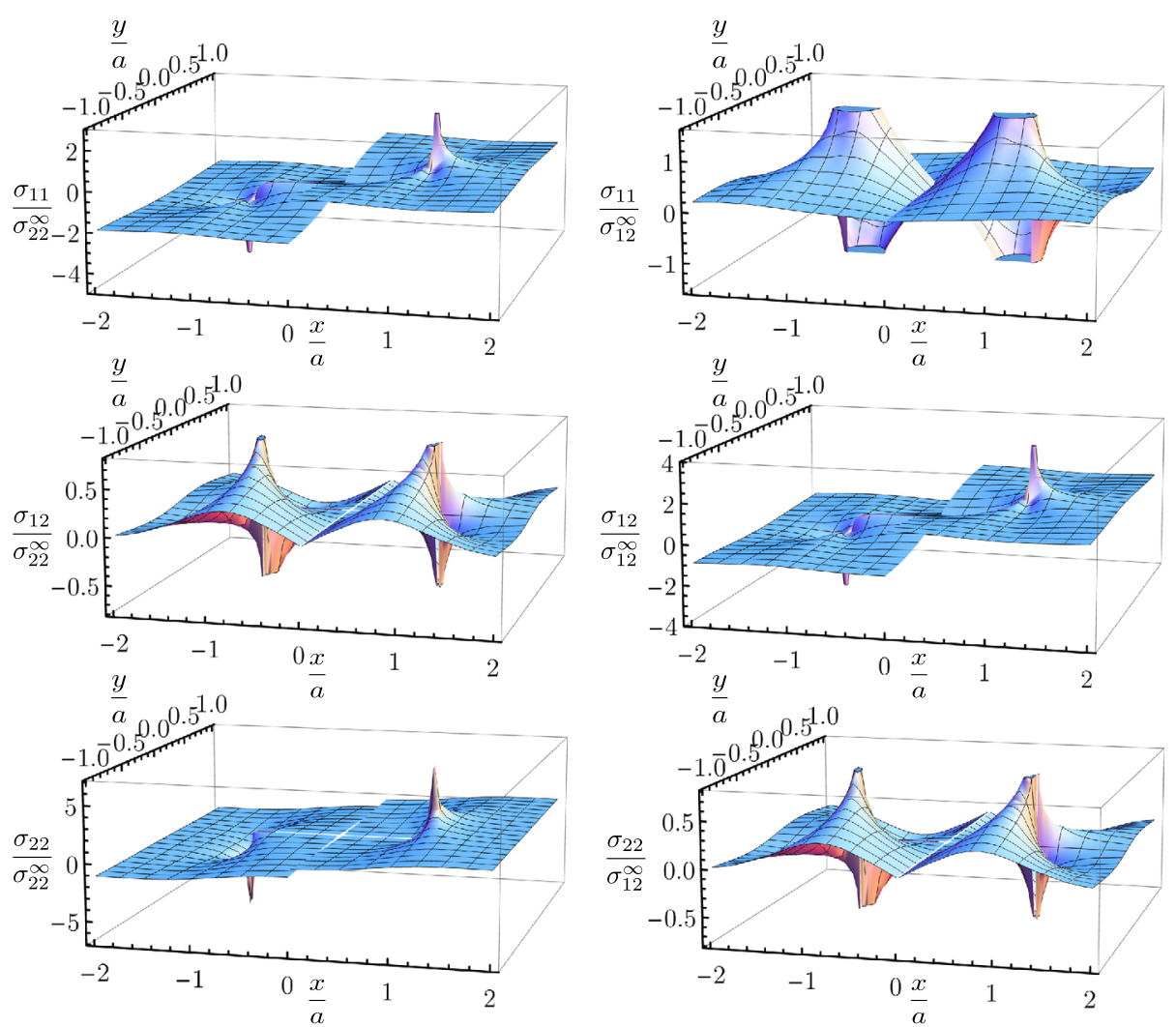

Fig. 3 Normalized stress fields for mode I (left) and mode II (right) for a Griffith crack calculated with the complex potentials of Eq. (9)

However, a majority of authors applies stress functions according to Westergaard's approach in terms of

$$
\begin{aligned}
Z_{I}(z) & =\frac{\sigma_{22}^{\infty} z}{\sqrt{z^{2}-a^{2}}}, \\
Z_{I I}(z) & =\frac{\sigma_{12}^{\infty} z}{\sqrt{z^{2}-a^{2}}},
\end{aligned}
$$

e.g., $[8,12,17,22,28,32,35,37]$ for mode I and [2, 9, 30, 31] for mixed mode crack opening, basically raising the same problems as the complex potentials of Eq. (9) in terms of symmetry and discontinuity. In the original paper by Westergaard [33], just the function $Z_{I}(z)$ is introduced, however, being dissimilar from the one in Eq. (14), actually reading

$$
Z_{I}(z)=\frac{\sigma_{22}^{\infty}}{\sqrt{1-\frac{a^{2}}{z^{2}}}} .
$$

While Eqs. (14) and (15) seem to be equivalent at the first glance, in fact they are not. This issue being one source of problems, the following section provides some detailed discussion. 


\section{The Problem of Complex Roots}

The Eqs. (9), (10), (14) and (15) include roots with complex radicands. The common root function $x \mapsto \sqrt{x}$ is defined for real $x \geq 0$ and results in non-negative values. In order to define the root function on $\mathbb{C}$ completely, the common polar form

$$
z=|z| \mathrm{e}^{\mathrm{i} \phi}
$$

is considered, with the argument $\phi \in(-\pi, \pi]$, and the root being

$$
\sqrt{z}=\sqrt{|z|} \mathrm{e}^{\frac{\mathrm{i} \phi}{2}} .
$$

Equation (17) is called the principal root and $\omega^{2}=z$ with unknown $\omega$ has the solutions $\omega= \pm \sqrt{z}$. Moreover, in Cartesian coordinates

$$
\sqrt{z}=\sqrt{x+\mathrm{i} y}= \begin{cases}\sqrt{\frac{|z|+x}{2}}+\mathrm{i} \sqrt{\frac{|z|-x}{2}}, & y \geq 0 \\ \sqrt{\frac{|z|+x}{2}}-\mathrm{i} \sqrt{\frac{|z|-x}{2}}, & y<0\end{cases}
$$

holds. Note that $z \mapsto \sqrt{z}$ is holomorphic in the slit plane $\mathbb{C} \backslash(-\infty, 0]$ and discontinuous in the real interval $(-\infty, 0)$. In general the principal branch is defined as

$$
z^{r}:=|z|^{r} \mathrm{e}^{r \mathrm{i} \phi}
$$

for $r \in \mathbb{R}$. It is straightforwardly shown that the derivation rule

$$
\frac{\mathrm{d}}{\mathrm{d} z}\left(z^{r}\right)=r z^{r-1}
$$

and the law of exponentiation

$$
z^{r_{1}} z^{r_{2}}=z^{r_{1}+r_{2}}
$$

hold in $\mathbb{C}$. In particular

$$
\begin{gathered}
z^{-r}=\frac{1}{z^{r}}, \\
z^{\frac{3}{2}}=(\sqrt{z})^{3},
\end{gathered}
$$

need to be considered in fracture mechanics problems. Note that with Eqs. (18) and (22), the complex potentials in Eq. (9) are formulated in Cartesian coordinates instead of employing polar coordinates. The irregularities in the complex potentials of the Griffith crack in Eqs. (9), (10) and (14) arise, because in general $z^{r} \omega^{r}$ does not equal $(z \omega)^{r}$. For the sake of clarification, the function

$$
f: \mathbb{C} \mapsto \mathbb{C}, \quad f(z)=\sqrt{z^{2}-a^{2}},
$$

appearing in these equations, will be compared to the function

$$
g: \mathbb{C} \mapsto \mathbb{C}, \quad g(z)=\sqrt{z-a} \sqrt{z+a},
$$


with its derivative

$$
g^{\prime}(z)=\frac{z}{\sqrt{z-a} \sqrt{z+a}}
$$

Furthermore,

$$
\overline{g(\bar{z})}=g(z)
$$

holds on $\mathbb{C} \backslash[-a, a]$, which is needed for the calculation of $\Psi^{\prime}$ in Eq. (10). Looking at

$$
\begin{aligned}
& f(-2 a)=\sqrt{3 a^{2}}=\sqrt{3} a, \\
& g(-2 a)=\sqrt{-3 a} \sqrt{-a}=-\sqrt{3} a,
\end{aligned}
$$

reveals that the functions do not coincide, even though they are treated equally in literature, starting with the cutting-edge work of Muskhelishvili [20], who replaces the initially introduced $g(z)$ by $f(z)$ in his derivation of Eq. (10). The function $f(z)$ even has discontinuities on the imaginary axis, which can be shown by the example

$$
\begin{aligned}
& \lim _{\varepsilon \searrow 0} f(\mathrm{i}+\varepsilon)=\lim _{\varepsilon \searrow 0} \sqrt{\underbrace{\varepsilon^{2}-1-a^{2}}_{<0}+2 \mathrm{i} \varepsilon}=+\mathrm{i} \sqrt{1+a^{2}}, \\
& \lim _{\varepsilon \searrow 0} f(\mathrm{i}-\varepsilon)=\lim _{\varepsilon \searrow 0} \sqrt{\underbrace{\varepsilon^{2}-1-a^{2}}_{<0}-2 \mathrm{i} \varepsilon}=-\mathrm{i} \sqrt{1+a^{2}} .
\end{aligned}
$$

It is hence not holomorphic in the relevant domain $\mathbb{C} \backslash[-a, a]$. In contrast, the function $g(z)$ of Eq. (24) is continuous on the imaginary axis and is equivalently represented by

$$
g(z)=z \sqrt{1-\frac{a^{2}}{z^{2}}} \quad \text { on } \quad \mathbb{C} \backslash[-a, a]
$$

which appears in Eq. (15). In order to prove this equivalence, the real arguments of $g(z)$ are examined.

\section{Proof}

Step 1: $x \in(a, \infty)$

$$
\Rightarrow g(x)=\sqrt{\underbrace{x-a}_{>0}} \sqrt{>0} \underbrace{x+a}_{>0}=\sqrt{x^{2}-a^{2}}=\sqrt{x^{2}} \sqrt{1-\frac{a^{2}}{x^{2}}}=x \sqrt{1-\frac{a^{2}}{x^{2}}}
$$

Step 2: As $g(z)=\sqrt{z+a} \sqrt{z-a}$ and $z \mapsto z \sqrt{1-\frac{a^{2}}{z^{2}}}$ are holomorphic on

$\mathbb{C} \backslash(-\infty, a]$ and equal on the continuum $(a, \infty)$, the identity

theorem provides the equivalence of Eqs. (29) and (24) on

$\mathbb{C} \backslash(-\infty, a]$. 
Step 3: $x \in(-\infty,-a)$

$$
\begin{aligned}
\Rightarrow g(x) & =\sqrt{\underbrace{x-a}_{<0}} \sqrt{<0} \underbrace{x+a}_{<0}=-\sqrt{a-x} \sqrt{-a-x}=-\sqrt{x^{2}-a^{2}} \\
& =-|x| \sqrt{1-\frac{a^{2}}{x^{2}}}=x \sqrt{1-\frac{a^{2}}{x^{2}}}
\end{aligned}
$$

Equation (29) shows that $g(z)$ is odd and holomorphic on $\mathbb{C} \backslash[-a, a]$. Finally, the behavior of $g(x)$, according to Eq. (24), is examined on the crack faces, i.e., $x \in[-a, a]$, where

$$
\begin{aligned}
\lim _{\varepsilon \searrow 0} g(x+\mathrm{i} \varepsilon) & =\lim _{\varepsilon \searrow 0} \sqrt{\underbrace{x-a}_{\leq 0}+\mathrm{i} \varepsilon} \sqrt{\underbrace{x+a}_{\geq 0}+\mathrm{i} \varepsilon} \\
& =\mathrm{i} \sqrt{a-x} \sqrt{a+x} \\
& =\mathrm{i} \sqrt{a^{2}-x^{2}} \\
& =g(x)
\end{aligned}
$$

and

$$
\begin{aligned}
\lim _{\varepsilon \searrow 0} g(x-\mathrm{i} \varepsilon) & =\lim _{\varepsilon \searrow 0}(-g(-x+\mathrm{i} \varepsilon)) \\
& =-g(-x) \\
& =-g(x)
\end{aligned}
$$

hold. The equivalence of Eqs. (24) and (29) is also valid for $x \in(0, a]$ where

$$
x \sqrt{1-\frac{a^{2}}{x^{2}}}=\mathrm{i} x \sqrt{\frac{a^{2}}{x^{2}}-1}=\mathrm{i} \sqrt{a^{2}-x^{2}}
$$

is satisfied. For $x \in(-a, 0]$ the equivalence is not given, as $g(x)$ in Eq. (24) is even on $[-a, a]$, whereas $x \mapsto x \sqrt{1-a^{2} / x^{2}}$ is odd. It is noteworthy that $f(z)$ and $g(z)$, Eqs. (23) and (24), are equal on $[-a, a]$ if $y=0$ is set without employing a limit.

To summarize, the relations

$$
\begin{aligned}
& \sqrt{z-a} \sqrt{z+a} \neq \sqrt{z^{2}-a^{2}} \quad \text { if } \operatorname{Re}[z]<0 \quad \text { and } \quad z \notin[-a, 0) \\
& \sqrt{z-a} \sqrt{z+a}=z \sqrt{1-\frac{a^{2}}{z^{2}}} \quad \text { on } \quad \mathbb{C} \backslash(-a, 0]
\end{aligned}
$$

are crucial for the deduction of complex potentials and holomorphic functions, respectively, of linear elastic crack problems. They emphasize that the function $\sqrt{z^{2}-a^{2}}$ must not be involved, which has been disregarded in Eqs. (9), (10) and (14), eventually giving rise to the problems depicted in Figs. 2 and 3. Equation (15), on the other hand, is correct. Equations (31) and (32) further demonstrate that approaching $y=0$ on the crack faces, necessarily involves taking an upper or lower limit. 
Fig. 4 Comparison of normalized stresses in the crack plane, i.e., $y=0$, for mode I loading from different sources

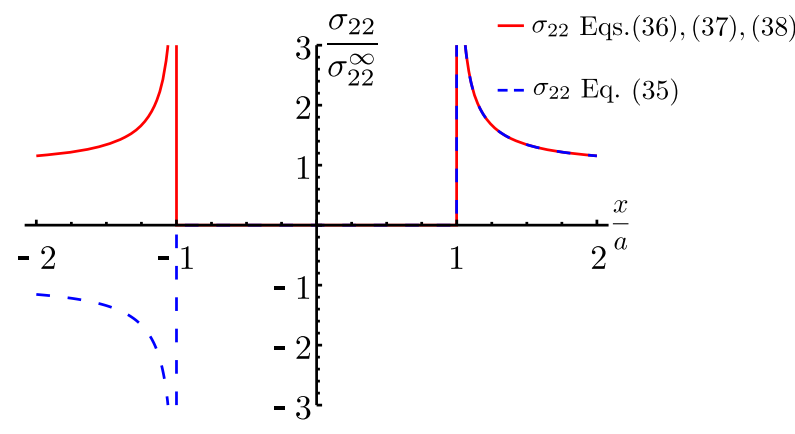

\section{Current Approaches Encountering the Deficiencies}

Despite of the deficiencies of the solutions illustrated in Figs. 2 and 3, stresses and displacements turn out to be correct in the positive half of the boundary value problem according to Fig. 1. To obtain crack fields on the whole domain from complex potentials being valid only for $\operatorname{Re}[z]>0$, a case distinction is commonly applied in literature. This is most suitably demonstrated taking the stress $\sigma_{22}$ in the crack plane, i.e., $y=0$, for a single mode I loading, which is given directly in $[10,16,24,38]$ and indirectly via Westergaard's stress functions according to Eq. (14) in [2, 9, 12, 17, 22, 28, 30-32, 35, 37]:

$$
\sigma_{22}(x)=\sigma_{22}^{\infty} \operatorname{Re}\left[\frac{x}{\sqrt{x^{2}-a^{2}}}\right] .
$$

$\sigma_{22}$ is obviously positive for $x>a$ and negative for $x<-a$. Therefore, the following formulation is intuitively employed

$$
\sigma_{22}(x)= \begin{cases}\sigma_{22}^{\infty} \operatorname{Re}\left[\frac{x}{\sqrt{x^{2}-a^{2}}}\right], & x \geq 0 \\ \sigma_{22}^{\infty} \operatorname{Re}\left[\frac{-x}{\sqrt{x^{2}-a^{2}}}\right], & x<0,\end{cases}
$$

actually lacking mathematical rigorousness. In, e.g., [4, 21] the stress is given monolithically as

$$
\sigma_{22}(x)=\sigma_{22}^{\infty} \operatorname{Re}\left[\frac{|x|}{\sqrt{x^{2}-a^{2}}}\right],
$$

however, the associated complex potentials are either not provided [21] or do not result in Eq. (37) [4]. Equation (15), yielding a mode I stress according to

$$
\sigma_{22}(x)=\sigma_{22}^{\infty} \operatorname{Re}\left[\frac{1}{\sqrt{1-\frac{a^{2}}{x^{2}}}}\right],
$$

is depicted in [14, 31], whereat [31], however, concurrently provide the stress function $Z_{I}(z)$ of Eq. (14). The stresses of the Eqs. (35) to (38) are illustrated in Fig. 4. 
In order to obtain Eq. (36), the complex potentials need to be formulated likewise by separating ranges of validity, for the example of $\Phi$ reading

$$
\Phi(z)= \begin{cases}-\frac{\sigma_{22}^{\infty}}{4} z+\frac{\sigma_{22}^{\infty}}{2} \sqrt{z^{2}-a^{2}}+\mathrm{i} \frac{\sigma_{12}^{\infty}}{2} z-\mathrm{i} \frac{\sigma_{12}^{\infty}}{2} \sqrt{z^{2}-a^{2}}, & \operatorname{Re}[z] \geq 0 \\ -\frac{\sigma_{22}^{\infty}}{4} z-\frac{\sigma_{22}^{\infty}}{2} \sqrt{z^{2}-a^{2}}+\mathrm{i} \frac{\sigma_{12}^{\infty}}{2} z+\mathrm{i} \frac{\sigma_{12}^{\infty}}{2} \sqrt{z^{2}-a^{2}}, & \operatorname{Re}[z]<0 .\end{cases}
$$

Avoiding a case distinction, monolithic complex potentials will be introduced in Sect. 6, where in particular the findings of Eq. (34) will play a crucial role.

Another approach compensating the limited validity of Eqs. (9) and Eq. (14) is found in, e.g., $[2,17,28,30]$. They employ the polar form of Westergaard's stress function in connection with the impermissible transformation $\sqrt{z^{2}-a^{2}}=\sqrt{z+a} \sqrt{z-a}$, see Eq. (34), thus changing the sign of the principal complex root, whereupon Eq. (14) yields

$$
Z_{I}(z)=\frac{\sigma_{22}^{\infty} z}{\sqrt{z-a} \sqrt{z+a}}=\frac{\sigma_{22}^{\infty} r}{\sqrt{r_{1} r_{2}}} \mathrm{e}^{\phi-\frac{1}{2} \phi_{1}-\frac{1}{2} \phi_{2}} .
$$

Equation (40) leads to correct crack fields in the whole domain, with $z=r \mathrm{e}^{\mathrm{i} \phi}, z-a=r_{1} \mathrm{e}^{\mathrm{i} \phi_{1}}$ and $z+a=r_{2} \mathrm{e}^{\mathrm{i} \phi_{2}}$, although the complex potential of Eq. (14) is only valid in the halfspace $\operatorname{Re}[z]>0$. The fact that an erroneous transformation eventually allows to obtain a comprehensive result from a constrained valid ansatz is one of the reasons, why the problems with the complex potentials or Westergaard stress functions have not yet attracted attention.

\section{Monolithic Complex Potentials}

In order to obtain the stress and displacement fields in the whole domain without any distinction of cases, the following complex potentials are introduced for $z \in \mathbb{C} \backslash[-a, a]$ :

$$
\begin{aligned}
\Phi(z)= & -\frac{\sigma_{22}^{\infty}}{4} z+\frac{\sigma_{22}^{\infty}}{2}(\sqrt{z-a} \sqrt{z+a})+\mathrm{i} \frac{\sigma_{12}^{\infty}}{2} z-\mathrm{i} \frac{\sigma_{12}^{\infty}}{2}(\sqrt{z-a} \sqrt{z+a}) \\
& +\frac{\sigma_{11}^{\infty}}{4} z-2 \mathrm{i} z \frac{\mu \varepsilon_{\infty}}{1+\kappa}+C, \\
\Phi^{\prime}(z)= & -\frac{\sigma_{22}^{\infty}}{4}+\frac{\sigma_{22}^{\infty}}{2} \frac{z}{\sqrt{z-a} \sqrt{z+a}}+\mathrm{i} \frac{\sigma_{12}^{\infty}}{2}-\mathrm{i} \frac{\sigma_{12}^{\infty}}{2} \frac{z}{\sqrt{z-a} \sqrt{z+a}} \\
& +\frac{\sigma_{11}^{\infty}}{4}-2 \mathrm{i} \frac{\mu \varepsilon_{\infty}}{1+\kappa}, \\
\Phi^{\prime \prime}(z)= & -\frac{\sigma_{22}^{\infty}}{2} \frac{a^{2}}{(z-a)^{\frac{3}{2}}(z+a)^{\frac{3}{2}}}+\mathrm{i} \frac{\sigma_{12}^{\infty}}{2} \frac{a^{2}}{(z-a)^{\frac{3}{2}}(z+a)^{\frac{3}{2}}}, \\
\Psi(z)= & \frac{\sigma_{22}^{\infty}}{2} z-\frac{\sigma_{22}^{\infty}}{2} \frac{a^{2}}{\sqrt{z-a} \sqrt{z+a}}+\frac{\mathrm{i} \sigma_{12}^{\infty}}{2} \frac{\left(2 z^{2}-a^{2}\right)}{\sqrt{z-a} \sqrt{z+a}}-\frac{\sigma_{11}^{\infty}}{2} z, \\
\Psi^{\prime}(z)= & \frac{\sigma_{22}^{\infty}}{2}+\frac{\sigma_{22}^{\infty}}{2} \frac{z a^{2}}{(z-a)^{\frac{3}{2}}(z+a)^{\frac{3}{2}}}+\frac{\mathrm{i} \sigma_{12}^{\infty}}{2} \frac{\left(2 z^{3}-3 z a^{2}\right)}{(z-a)^{\frac{3}{2}}(z+a)^{\frac{3}{2}}}-\frac{\sigma_{11}^{\infty}}{2} .
\end{aligned}
$$



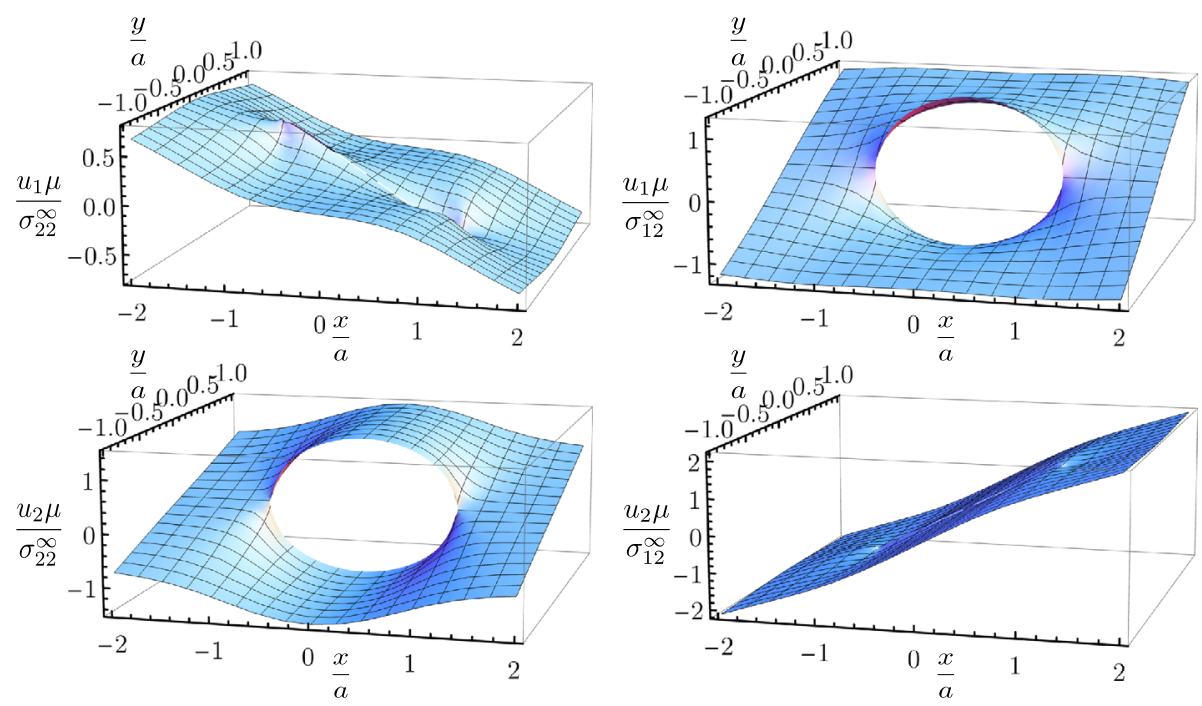

Fig. 5 Normalized displacement fields for mode I (left) and mode II (right) for a Griffith crack calculated with the complex potentials of Eq. (41)

For the sake of generality, an integration constant $C=C_{1}+\mathrm{i} C_{2}$ in $\Phi(z)$ accounts for linear rigid body motion and the last term in $\Phi^{\prime}(z)$ incorporates rigid body rotation. While translational motion vanishes in the local crack related coordinate system, trivially satisfying the condition

$$
u_{i}^{+}(0)+u_{i}^{-}(0)=0 \quad, \quad i=1,2,
$$

and thus $C=0$, the rotational motion does not basically vanish, depending on the boundary conditions. In $[19,20]$ it is introduced in "general formulae", however, disregarded in the specific boundary value problem.

The rotational term introduced in Eq. (41) has to satisfy the condition of rigid body rotation at infinity, i.e.,

$$
\varepsilon_{\infty}=\lim _{|z| \rightarrow \infty} \frac{1}{2}\left(\frac{\partial u_{1}(z)}{\partial y}-\frac{\partial u_{2}(z)}{\partial x}\right)
$$

which, with the displacement gradients of Eq. (5), leads to $-2 \mathrm{i} \mu \varepsilon_{\infty} /(1+\kappa)$ in $\Phi^{\prime}$. According to Eq. (4), the stress fields are not affected by the additional term. Inserting Eq. (41) into Eq. (4), with $\sigma_{11}^{\infty}=0$ and zero rigid body rotation, the crack fields depicted in Figs. 5 and 6 are obtained, consistently employing the principal complex root. Due to expected symmetries as well as continuities and discontinuities, the plots illustrate the plausibility of the results. 

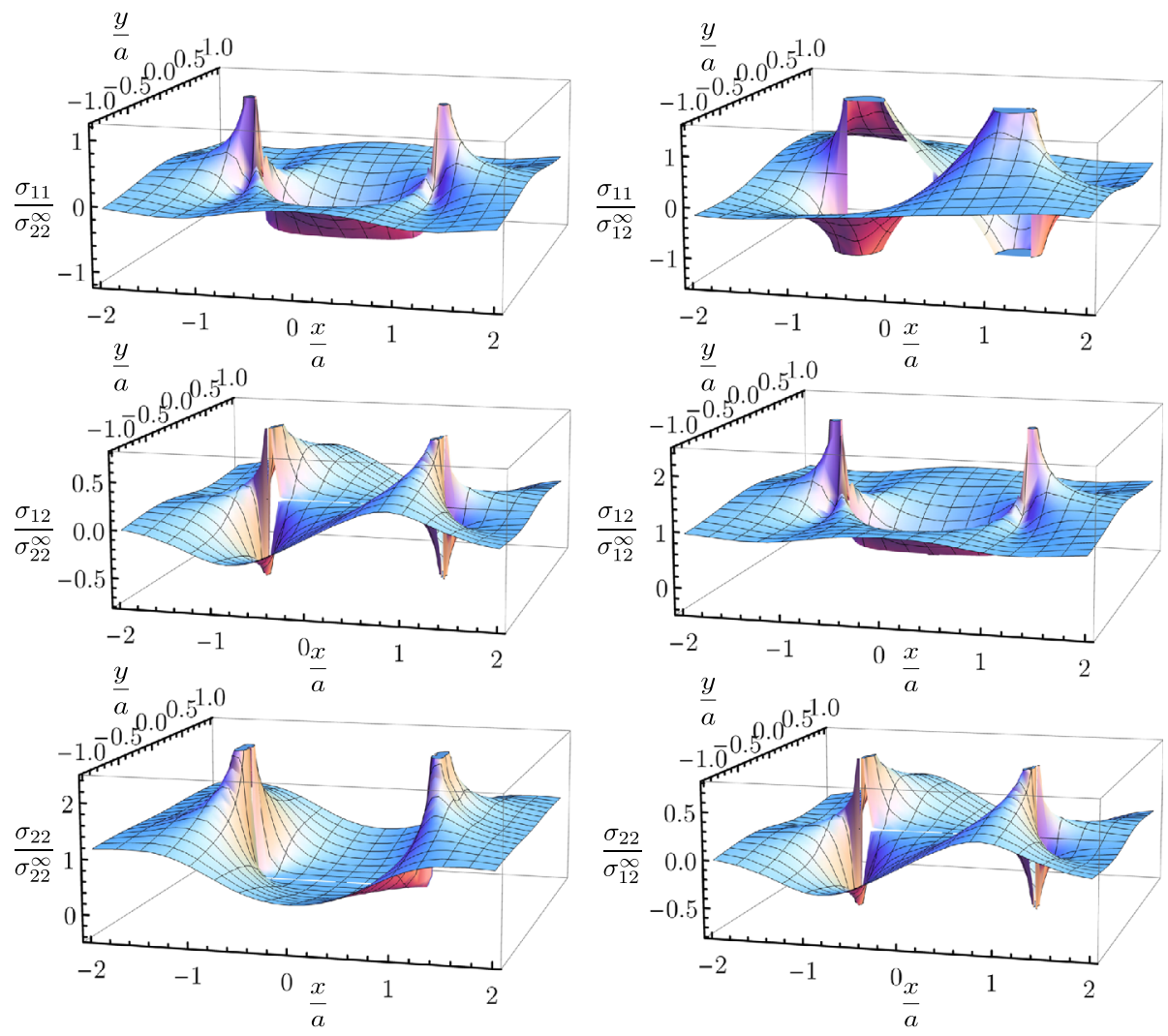

Fig. 6 Normalized stress fields for mode I (left) and mode II (right) for a Griffith crack calculated with the complex potentials of Eq. (41)

Equations (24) and (29) being equivalent for $z \in \mathbb{C} \backslash(-a, 0]$, the complex potentials alternatively are formulated as follows:

$$
\begin{aligned}
\Phi(z)= & -\frac{\sigma_{22}^{\infty}}{4} z+\frac{\sigma_{22}^{\infty}}{2}\left(z \sqrt{1-\frac{a^{2}}{z^{2}}}\right)+\mathrm{i} \frac{\sigma_{12}^{\infty}}{2} z-\mathrm{i} \frac{\sigma_{12}^{\infty}}{2}\left(z \sqrt{1-\frac{a^{2}}{z^{2}}}\right) \\
& +\frac{\sigma_{11}^{\infty}}{4} z-2 \mathrm{i} z \frac{\mu \varepsilon_{\infty}}{1+\kappa}, \\
\Psi(z)= & \frac{\sigma_{22}^{\infty}}{2} z-\frac{\sigma_{22}^{\infty}}{2} \frac{a^{2}}{z \sqrt{1-\frac{a^{2}}{z^{2}}}}+\frac{\mathrm{i} \sigma_{12}^{\infty}}{2} \frac{\left(2 z-\frac{a^{2}}{z}\right)}{\sqrt{1-\frac{a^{2}}{z^{2}}}}-\frac{\sigma_{11}^{\infty}}{2} z .
\end{aligned}
$$

In the crack plane $(y \rightarrow \pm 0)$, being of major interest in fracture mechanics, the holomorphic functions of Eqs. (41) or (44) for $\sigma_{11}^{\infty}=0$ and $\varepsilon_{\infty}=0$ yield the following results for 
mode I,

$$
\begin{aligned}
& \sigma_{11}(x)=\sigma_{22}^{\infty} \operatorname{Re}\left[\frac{x}{\sqrt{x+a} \sqrt{x-a}}-1\right]= \begin{cases}-\sigma_{22}^{\infty}, & |x|<a \\
\frac{\sigma_{22}^{\infty}}{\sqrt{1-\frac{a^{2}}{x^{2}}}}-\sigma_{22}^{\infty}, & |x|>a, \\
& \end{cases} \\
& \sigma_{12}(x)=0, \\
& \sigma_{22}(x)=\sigma_{22}^{\infty} \operatorname{Re}\left[\frac{x}{\sqrt{x+a} \sqrt{x-a}}\right]= \begin{cases}0, & |x|<a \\
\frac{\sigma_{22}^{\infty}}{\sqrt{1-\frac{a^{2}}{x^{2}}}}, & |x|>a,\end{cases} \\
& u_{1}(x)=\operatorname{Re}\left[\sigma_{22}^{\infty}\left(-\frac{\kappa+1}{8 \mu} x+\frac{\kappa-1}{4 \mu} \sqrt{x+a} \sqrt{x-a}\right)\right] \\
& = \begin{cases}-\frac{\kappa+1}{8 \mu} \sigma_{22}^{\infty} x, & |x| \leq a \\
\sigma_{22}^{\infty}\left(-\frac{\kappa+1}{8 \mu} x+\frac{\kappa-1}{4 \mu} x \sqrt{\left.1-\frac{a^{2}}{x^{2}}\right),}\right. & |x|>a,\end{cases} \\
& u_{2}^{ \pm}(x)= \pm \frac{\kappa+1}{4 \mu} \operatorname{Im}[\sqrt{x-a} \sqrt{x+a}]= \begin{cases} \pm \frac{\kappa+1}{4 \mu} \sqrt{a^{2}-x^{2}} \sigma_{22}^{\infty}, & |x|<a \\
0, & |x| \geq a,\end{cases}
\end{aligned}
$$

and for mode II

$$
\begin{aligned}
& \sigma_{11}^{ \pm}(x)= \pm \sigma_{12}^{\infty} \operatorname{Im}\left[\frac{2 x}{\sqrt{x+a} \sqrt{x-a}}\right]= \begin{cases}\mp \frac{2 x \sigma_{12}^{\infty}}{\sqrt{a^{2}-x^{2}}}, & |x|<a \\
0, & |x|>a,\end{cases} \\
& \begin{aligned}
\sigma_{12}(x) & =\sigma_{12}^{\infty} \operatorname{Re}\left[\frac{x}{\sqrt{x+a} \sqrt{x-a}}\right]= \begin{cases}0, & |x|<a \\
\frac{\sigma_{12}^{\infty}}{\sqrt{1-\frac{a^{2}}{x^{2}}},} & |x|>a,\end{cases} \\
\sigma_{22}(x) & =0, \\
u_{1}^{ \pm}(x) & = \pm \frac{\kappa+1}{4 \mu} \operatorname{Im}[\sqrt{x-a} \sqrt{x+a}] \\
& = \begin{cases} \pm \frac{\kappa+1}{4 \mu} \sqrt{a^{2}-x^{2}} \sigma_{12}^{\infty}, & |x|<a \\
0, & |x| \geq a,\end{cases} \\
u_{2}(x)= & \operatorname{Re}\left[\sigma_{12}^{\infty}\left(\frac{\kappa+1}{4 \mu} x-\frac{\kappa-1}{4 \mu} \sqrt{x+a} \sqrt{x-a}\right)\right]
\end{aligned}
\end{aligned}
$$




$$
= \begin{cases}\frac{\kappa+1}{4 \mu} \sigma_{12}^{\infty} x, & |x| \leq a \\ \sigma_{12}^{\infty}\left(\frac{\kappa+1}{4 \mu} x-\frac{\kappa-1}{4 \mu} x \sqrt{1-\frac{a^{2}}{x^{2}}}\right), & |x|>a .\end{cases}
$$

Here, \pm and $\mp$ indicate the positive $(y \searrow 0)$ or negative $(y \nearrow 0)$ crack face. Note that the complex potentials in the formulation of Eq. (41) yield the result of the positive crack face if $y=0$ is inserted without employing an appropriate limit. The complex potentials in Eq. (44) likewise account for the positive crack face for $x>0$ and the negative crack face for $x<0$. Taking an upper or lower limit of $y \rightarrow 0$ therefore is vital to distinguish between the crack faces and to obtain appropriate solutions.

With Eq. (13) and assuming $\varepsilon_{\infty}=0, \Phi^{\prime}(z)$ and $\Psi^{\prime}(z)$ of Eq. (41) are obtained from the relations

$$
\begin{aligned}
& \Phi^{\prime}(z)=\frac{\left(2 \Gamma+\overline{\Gamma_{2}}\right) z}{2 \sqrt{z-a} \sqrt{z+a}}-\frac{1}{2} \overline{\Gamma_{2}}, \\
& \Omega(z)=\frac{\left(2 \Gamma+\overline{\Gamma_{2}}\right) z}{2 \sqrt{z-a} \sqrt{z+a}}+\frac{1}{2} \overline{\Gamma_{2}},
\end{aligned}
$$

providing the counterpart of Eq. (10), however, not being equivalent, bearing in mind that $f(z) \neq g(z)$, according to Eq. (34). Introducing a function $h(x)$ on the crack faces with $x \in(-a, a)$ as the limit $y \rightarrow 0$ from above of $\Phi^{\prime}(z)+\overline{\Gamma_{2}} / 2$ in Eq. (47), i.e.,

$$
\begin{aligned}
h(x) & =\lim _{\varepsilon \searrow 0}\left(\Phi^{\prime}(x+\varepsilon \mathrm{i})+\frac{1}{2} \overline{\Gamma_{2}}\right) \\
& =\lim _{\varepsilon \searrow 0} \frac{2 \Gamma+\overline{\Gamma_{2}}}{2} \frac{x+\varepsilon \mathrm{i}}{\sqrt{x+\varepsilon i-a} \sqrt{x+\varepsilon i+a}} \\
& =\frac{2 \Gamma+\overline{\Gamma_{2}}}{2 \mathrm{i}} \frac{x}{\sqrt{a^{2}-x^{2}}} \quad, \quad x \in(-a, a),
\end{aligned}
$$

the condition

$$
\int_{-a}^{a} h(x) \mathrm{d} x=0
$$

postulated in [20] is still satisfied as $h(x)$ is odd.

\section{Conclusion}

Complex potentials and holomorphic functions, respectively, for a crack in an infinite elastic domain under mixed-mode loading are provided. In contrast to approaches commonly taken from literature, stresses and displacements in the whole domain are displayed without unphysical discontinuities or point symmetry, furthermore allowing for arbitrary rigid body rotation. Basically, problems arise from misinterpretation of roots in the complex plane. This issue, so far, didn't attract attention, probably for two reasons. Firstly, fracture mechanics applications focus on merely a few aspects of the crack solution, restricted to the crack faces 
and the ligament of just one crack tip. Secondly, a rigorous mathematical derivation is not required to obtain a comprehensive solution, but can be replaced by intuitive continuation of a partially valid solution. Finally it is noteworthy, that the addressed problems of the complex potentials or Westergaard stress functions do not only arise for the Griffith crack, but probably for various other crack problems, e.g., the Dugdale crack model $[3,5,11,31]$.

Funding Note Open Access funding enabled and organized by Projekt DEAL.

Availability of Data and Material Not applicable.

Code Availability Not applicable.

\section{Declarations}

Conflict of Interest The authors declare that they have no conflict of interest.

Open Access This article is licensed under a Creative Commons Attribution 4.0 International License, which permits use, sharing, adaptation, distribution and reproduction in any medium or format, as long as you give appropriate credit to the original author(s) and the source, provide a link to the Creative Commons licence, and indicate if changes were made. The images or other third party material in this article are included in the article's Creative Commons licence, unless indicated otherwise in a credit line to the material. If material is not included in the article's Creative Commons licence and your intended use is not permitted by statutory regulation or exceeds the permitted use, you will need to obtain permission directly from the copyright holder. To view a copy of this licence, visit http://creativecommons.org/licenses/by/4.0/.

\section{References}

1. Airy, G.B.: On the Strains in the Interior of Beams. In Report of the thirty-second Meeting of the British Association for the Advancement of Science pp. 82-86. Report of the British Association for the Advancement of Science (1862)

2. Atkinson, B.K.: Fracture Mechanics of Rock. Academic Press, London,UK (1987)

3. Becker, W., Gross, D.: About the Dugdale crack under mixed mode loading. Int. J. Fract. 37(3), 163-170 (1988)

4. Benthem, J., Koiter, W.: Asymptotic approximations to crack problems. In: Sih, G. (ed.) Mechanics of Fracture: Methods of Analysis and Solutions of Crack Problems, vol. 1, Chap. 3, pp. 131-178. Springer, Dordrecht (1973)

5. Burdekin, F.M., Stone, D.: The crack opening displacement approach to fracture mechanics in yielding materials. J. Strain Anal. 1(2), 145-153 (1966)

6. Eftis, J., Subramonian, N.: Crack border stress and displacement equations revisited. Eng. Fract. Mech. 9, 189-210 (1977)

7. Eftis, J., Subramonian, N.: The inclined crack under biaxial load. Eng. Fract. Mech. 10, $43-67$ (1978)

8. England, A., Green, A.: Some two-dimensional punch and crack problems in classical elasticity. In: Mathematical Proceedings of the Cambridge Philosophical Society, vol. 59, pp. 489-500. Cambridge University Press, Cambridge (1963)

9. Gdoutos, E.E.: Fracture Mechanics: An Introduction, vol. 3. Springer, Cham (2020)

10. Gross, D., Seelig, T.: Fracture Mechanics: With an Introduction to Micromechanics, vol. 3. Springer, Berlin (2017)

11. Hayes, D., Williams, J.: A practical method for determining Dugdale model solutions for cracked bodies of arbitrary shape. Int. J. Fract. Mech. 8(3), 239-256 (1972)

12. Heng, Z., McCammond, D., Tabarrok, B.: A development in the use of Westergaard stress functions for the solution of Griffith crack problems. Trans. Can. Soc. Mech. Eng. 8(3), 142-145 (1984)

13. Inglis, C.E.: Stresses in a plate due to the presence of cracks and sharp corners. Trans. Inst. Nav. Archit. 55, 219-241 (1913)

14. Irwin, G.R.: Analysis of stresses and strains near the end of a crack traversing a plate. J. Appl. Mech. 24, 361-364 (1957)

15. Kolosov, G.V.: On an Application of Complex Function Theory to a Plane Problem of the Mathematical Theory of Elasticity. Yuriev (1909). (in Russian) 
16. Kuna, M.: Finite Elements in Fracture Mechanics. Springer, Berlin (2013)

17. Kundu, T.: Fundamentals of Fracture Mechanics. CRC Press, Boca Raton (2008)

18. Love, A.E.H.: A Treatise on the Mathematical Theory of Elasticity. Cambridge University Press, Cambridge (1927)

19. Muskhelishvili, N.I.: Singular Integral Equations: Boundary Problems of Function Theory and Their Application to Mathematical Physics. Noordhoff, Groningen (1953)

20. Muskhelishvili, N.I.: Some Basic Problems of the Mathematical Theory of Elasticity. Noordhoff, Groningen (1953). (published in Russian in 1933)

21. Panasyuk, V.V.: Limiting equilibrium of brittle solids with fractures. In: Tech. Rep., Air Force Systems Command Wright-Patterson AFB OH Foreign Technology Division (1969)

22. Perez, N.: Fracture Mechanics. Springer, Berlin (2017)

23. Rice, J.: Mathematical analysis in the mechanics of fracture. In: Liebowitz, H. (ed.) Fracture: An Advanced Treatise, vol. 2, Chap. 3, pp. 191-311. Academic Press, N.Y. (1968)

24. Richard, H.A., Sander, M.: Ermüdungsrisse: Erkennen, sicher beurteilen, vermeiden (2009). Vieweg+ Teubner Verlag/GWV Fachverlage GmbH

25. Sanford, R.J.: A critical re-examination of the Westergaard method for solving opening mode crack problems. Mech. Res. Commun. 6, 289-294 (1979)

26. Shi, Z.: Crack Analysis in Structural Concrete: Theory and Applications. Butterworth-Heinemann, Stoneham (2009)

27. Sih, G.: On the Westergaard method of crack analysis. Int. J. Fract. Mech. 2(4), 628-631 (1966)

28. Sneddon, I.N.: The distribution of stress in the neighbourhood of a crack in an elastic solid. Proc. R. Soc. Lond. Ser. A, Math. Phys. Sci. 187(1009), 229-260 (1946)

29. Sun, C., Farris, T.: On the completeness of the Westergaard stress functions. Int. J. Fract. 40(1), 73-77 (1989)

30. Sun, C., Jin, Z.H.: Fracture Mechanics. Academic Press, Boston (2012)

31. Tada, H., Paris, P.C., Irwin, G.R.: The Stress Analysis of Cracks Handbook, vol. 2. ASME Press, New York (2000)

32. Theocaris, P.S., Michopoulos, J.G.: A closed-form solution of a slant crack under biaxial loading. Eng. Fract. Mech. 17, 97-123 (1983)

33. Westergaard, H.M.: Bearing pressures and cracks. J. Appl. Mech. 6, 49-53 (1939)

34. Wieghardt, K.: Über das Spalten und Zerreißen elastischer Körper. Z. Math. Phys. 55(1), 2 (1907)

35. Willmore, T.: The distribution of stress in the neighbourhood of a crack. Q. J. Mech. Appl. Math. 2(1), 53-63 (1949)

36. Wirtinger, W.: Zur formalen Theorie der Funktionen von mehr komplexen Veränderlichen. Math. Ann. 97(1), 357-375 (1927)

37. Yoffe, E.H.: LXXV. The moving Griffith crack. Lond., Edinb., Dublin Philos. Mag. J. Sci. 42(330), 739-750 (1951)

38. Zehnder, A.T.: Lecture Notes on Fracture Mechanics, vol. 20, p. 22. Cornell University Press, Ithaca (2007)

Publisher's Note Springer Nature remains neutral with regard to jurisdictional claims in published maps and institutional affiliations. 